\title{
CASCII Overexpression Predicts Poor Prognosis and Regulates Cell Proliferation and Apoptosis in Ovarian Carcinoma
}

This article was published in the following Dove Press journal: Cancer Management and Research

\section{Ying Cui \\ Guihua Shen \\ Dan Zhou \\ Fengli Wu}

Department of Gynecology, Beijing Hospital, National Center of Gerontology, Institute of Geriatric Medicine, Chinese Academy of Medical Sciences, Beijing City 100730, People's Republic of China
Correspondence: Ying Cui Department of Gynecology, Beijing Hospital, National Center of Gerontology, Institute of Geriatric Medicine, Chinese Academy of Medical Sciences, No. I Dahua Road, Dongcheng District, Beijing City 100730, People's

Republic of China 100730

Email uvh1528@163.com
Introduction: It is known that CASC11 can promote colorectal cancer. However, the function of CASC11 in ovarian carcinoma (OC) remains elusive.

Methods: In this study, we measured the expression levels of CASC11 and miR-182 in both OC and healthy control samples by performing qPCR. The interaction between CASC11 and miR-182 was analyzed by the overexpression experiment and qPCR. Cell apoptosis was analyzed by cell apoptosis assay, and the prognostic value of CASC11 for OC was analyzed by survival curve analysis.

Results: We found that CASC11 and microRNA-182 (miRNA-182) were upregulated in OC. Plasma CASC11 was upregulated in OC patients and predicted early-stage OC. Follow-up study revealed that high plasma levels of CASC11 were closely correlated with poor survival conditions of OC patients. CASC11 and miRNA-182 were positively correlated in OC. Overexpression of CASC11 mediated the upregulation of miRNA-182 in cells of OC cell lines, while miRNA-182 overexpression did not significantly affect CASC11 expression. Overexpression of CASC11 and miRNA-182 promoted cancer cell proliferation and inhibited cancer cell apoptosis.

Conclusion: Therefore, CASC11 overexpression predicts poor prognosis and CASC11 regulates cell proliferation and apoptosis as well as microRNA-182 expression in ovarian carcinoma.

Keywords: ovarian carcinoma, CASC11, miRNA-182, proliferation, apoptosis

\section{Introduction}

Ovarian carcinoma (OC) is one of the most frequently diagnosed cancers among females and it causes high mortality rate. ${ }^{1}$ In the United States, OC affects more than 20,000 new cases and is responsible for about 14,000 deaths every year. ${ }^{2}$ Surgical resection is the only radical treatment for OC patients. However, the application of surgery is challenged by the existence of tumor metastasis in most cases by the time of diagnosis. ${ }^{3}$ Therefore, the overall 5-year survival rate of OC patients is below $30 \%$ in many countries of the world. ${ }^{3-5}$ It is generally believed that early diagnosis followed by proper treatment is the key for the survival of OC patients.

Long non-coding RNAs are a group of non-protein-coding RNAs composed of more than 200 nucleotides. ${ }^{6}$ Studies in the past several decades have shown that lncRNAs are key players in many critical biological processes, such as cell differentiation and development. ${ }^{7}$ Besides that, lncRNAs are also key players in human diseases, including cancers. $^{8,9}$ It has been well established that lncRNAs may interact with microRNAs (miRNAs) to achieve its biological roles. ${ }^{10}$ LncRNA cancer susceptibility candidate 11 
(CASC11) is a recently identified oncogenic lncRNA in colorectal cancer. ${ }^{11}$ CASC11 is located on chromosome 8. After transcription, CASC11 directly target heterogeneous ribonucleoprotein $\mathrm{K}$ to activate $\mathrm{WNT} / \beta$-catenin, which in turn regulates cancer cell behaviors. ${ }^{11}$ MicroRNA-182 (chromosome 7) is overexpressed in $\mathrm{OC}$ and interacts with programmed cell death 4 (PDCD4) to promote OC cell invasion and proliferation. ${ }^{12}$ Our preliminary microarray data showed that CASC11 and microRNA-182 were positively correlated in OC. This study was therefore carried out to investigate the potential interaction between CASC11 and microRNA-182 in OC.

\section{Materials and Methods}

\section{Human Materials and Cell Lines}

Tumor tissues as well as paired healthy tissue within about $2 \mathrm{~cm}$ around the tumor were collected from 64 patients with OC (serous epithelial OC, 28 to 61 years, $44.4 \pm 5.4$ years) through MRI-guided fine needle biopsies. All tissue samples were confirmed by histopathological exams: tumor tissues contained more than $95 \%$ cancer cells and healthy tissues contained no cancer cells. Plasma specimens were obtained from these $64 \mathrm{OC}$ patients and 58 healthy volunteers (29 to 63 years, $44.9 \pm 5.1$ years). Patients included 8 cases at AJCC (the same as FIGO stage) stage I, 9 in stage II, 7 in stage III and 40 in stage IV. These participants were admitted by Beijing Hospital Gynecology from January 2011 to January 2013. Inclusion criteria of patients: 1) patients diagnosed with OC through pathological examinations; 2) patients willing to participate; 3) patients completed treatment and 5-year follow-up. Exclusion criteria: 1) patients complicated with other diseases; 2) treatment was performed before admission; 3) patients died of other causes during follow-up. There were no significant differences in age, body mass index (BMI) and other basic background information between the two groups. This study passed the review of Ethics Committee of Beijing Hospital Gynecology. All participants signed informed consent before admission.

Cells of UWB1.289 and UWB1.289+BRCA1 OC cell lines (ATCC, USA) were used in this study. Cell culture and subculture were performed following the manufacturer's instructions.

\section{Follow-Up}

All patients were followed-up for 5 years after discharge. The overall survival conditions and causes of deaths were recorded.

\section{RT-qPCR}

To detectCASC11 and miRNA-182, total RNA and miRNA extractions were performed using RNAzol ${ }^{\circledR}$ RT RNA Isolation Reagent (Sigma-Aldrich) and mirVana miRNA Isolation Kit (Thermo Fisher Scientific), respectively. Reverse transcriptions were performed using AMV Reverse Transcriptase (NEB, USA) and TaqMan MicroRNA Reverse Transcription Kit (Thermo Fisher Scientific), respectively. In total RNA and miRNA reverse transcriptions, $1.2 \mathrm{ug}$ total RNAs and $100 \mathrm{ng}$ miRNAs were used in $20 \mathrm{ul} \mathrm{system.} \mathrm{Luna}{ }^{\circledR}$ Universal One-Step RT-qPCR Kit (NEB) and TaqMan MicroRNA Reverse Transcription Kit (Thermo Fisher Scientific) were used to prepare PCR mixtures. $1 \mathrm{ul}$ cDNA was added into $20 \mathrm{ul} \mathrm{PCR}$ reaction system. Expression of CASC11 and miRNA-182 was normalized using $2^{-\Delta \Delta C T}$ method.

\section{Cell Transfection}

CASC11 cDNA-expressing pIRSE2 vectors and empty pIRSE2 vectors were constructed by Sangon (Shanghai, China). miRNA-182 mimic and negative control miRNAs were from Sigma-Aldrich. UWB1.289 and UWB1.289 +BRCA1 OC cells were collected at $70-80 \%$ confluence and transfections were performed using Lipofectamine 2000 reagent (Thermo Fisher Scientific) with vectors and miRNAs at doses of 10 and $50 \mathrm{nM}$, respectively. Cells were incubated with the transfection mixture for $5 \mathrm{~h}$, followed by incubation in fresh cell culture medium before the subsequent experiments. Negative control miRNA and empty vector transfections were negative control cells. Cells without any treatments were control cells.

\section{Cell Proliferation Assay}

Cell proliferation abilities were detected at this time point. Briefly, $5 \times 10^{4}$ cells were mixed with $1 \mathrm{~mL}$ cell culture medium to prepare single-cell suspensions. 96-well plates were used to cultivate cells $(0.1 \mathrm{~mL}$ cell suspension per well) under conditions of $37^{\circ} \mathrm{C}$ and $5 \% \mathrm{CO}_{2}$ ). CCK-8 solution (10ul, Sigma-Aldrich) was added at $4 \mathrm{~h}$ before harvesting cells. Cells were harvested every $24 \mathrm{~h}$ until 96 h. Following the addition of 10ul DMSO, OD values were measured at $450 \mathrm{nM}$.

\section{Cell Apoptosis Assay}

Briefly, $1 \mathrm{~mL}$ serum-free cell culture medium was mixed with $5 \times 10^{4}$ cells to make single-cell suspension. A 6 -well plate was used to cultivate cells ( $2 \mathrm{~mL}$ per well) for $48 \mathrm{~h}$. After that 
cells were digested with $0.25 \%$ trypsin. After staining with and propidium iodide (PI) and Annexin V-FITC (Dojindo, Japan), flow cytometry was performed to separate cells.

\section{Statistical Analysis}

All data were mean values from 3 biological replicates. SPSS19.0 (SPSS Inc., Chicago, IL, USA) was used to carry all statistical analyses. Differences between the two groups of participants were explored by unpaired $t$-test. Differences between two types of tissues from the patient group were explored by paired $t$-test. ANOVA (one-way) and Tukey's test were used to explore differences among multiple groups. Diagnostic analysis was performed by ROC curve analysis. Correlations were analyzed by Pearson's correlation coefficient. OC patients were divided
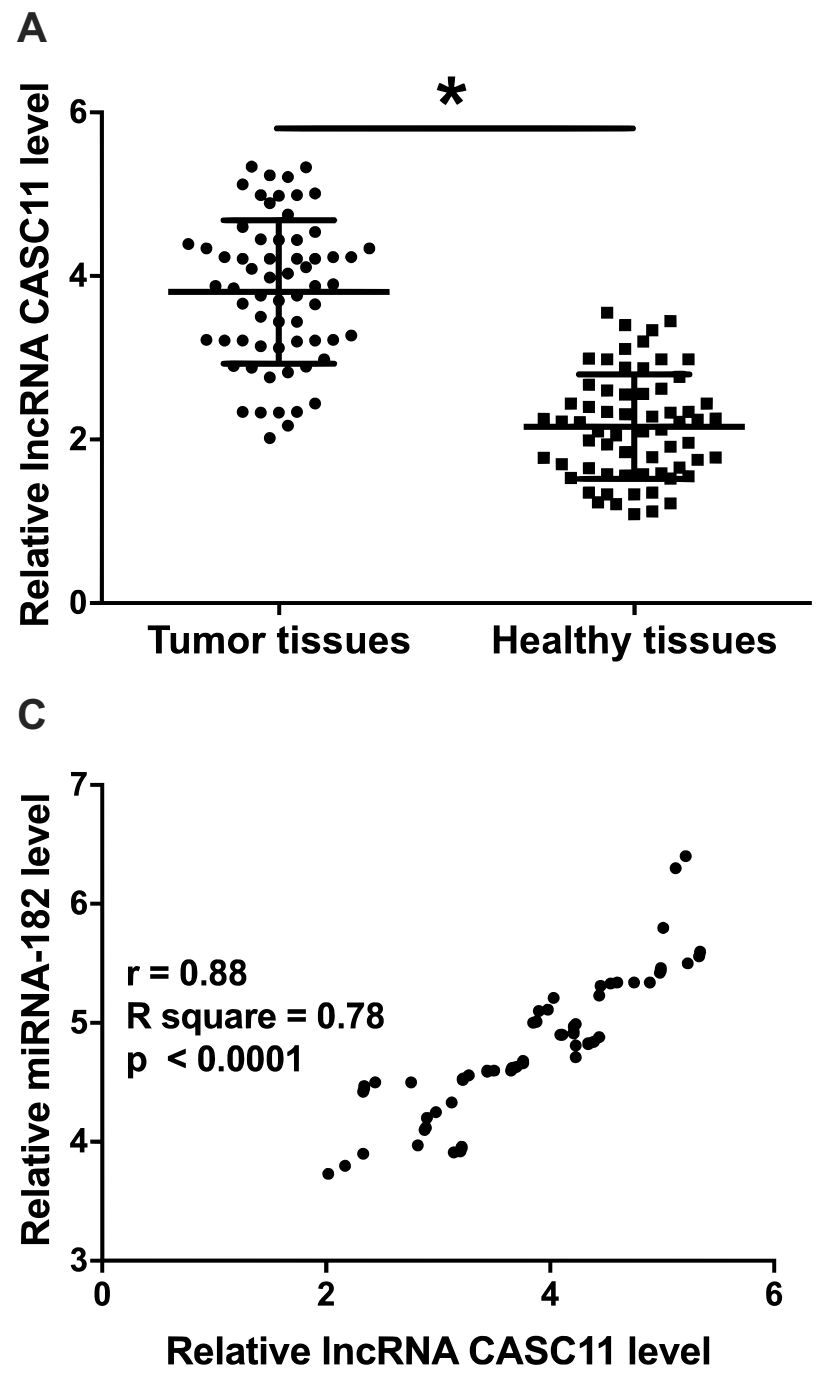

into high $(\mathrm{n}=34)$ and low $(\mathrm{n}=30)$ CASC11 level groups according to Youden's index and survival curves were plotted and compared by Kaplan-Meier and log-rank test, respectively. $\mathrm{p}<0.05$ was statistically significant.

\section{Results}

Expression Levels of CASCII and miRNA-I82 Were Upregulated in Tumor Tissues and Positively Correlated

Results of RT-qPCR showed that, compared to healthy tissues, expression of CASC11 (Figure 1A) and miRNA182 (Figure 1B) was significantly upregulated in tumor tissues $(\mathrm{p}<0.05)$. Correlation analysis showed that, in tumor tissues, CASC11 and miRNA-182 were positively

B

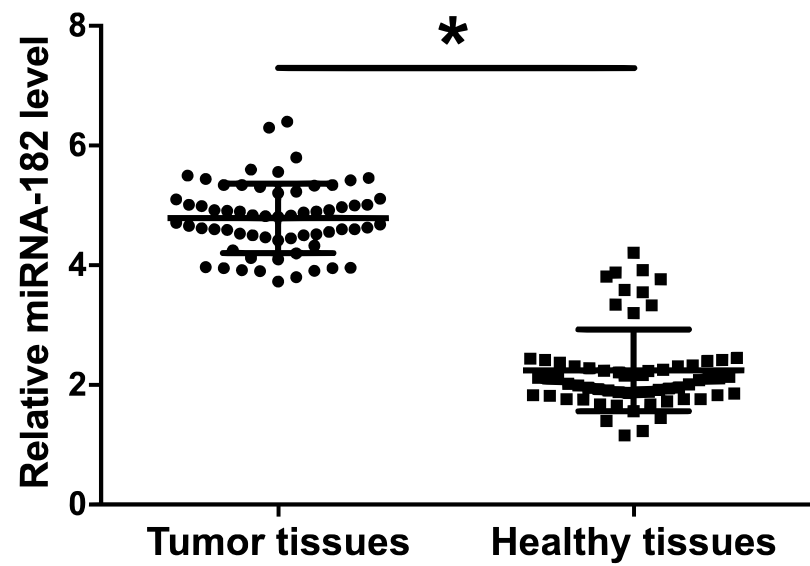

D

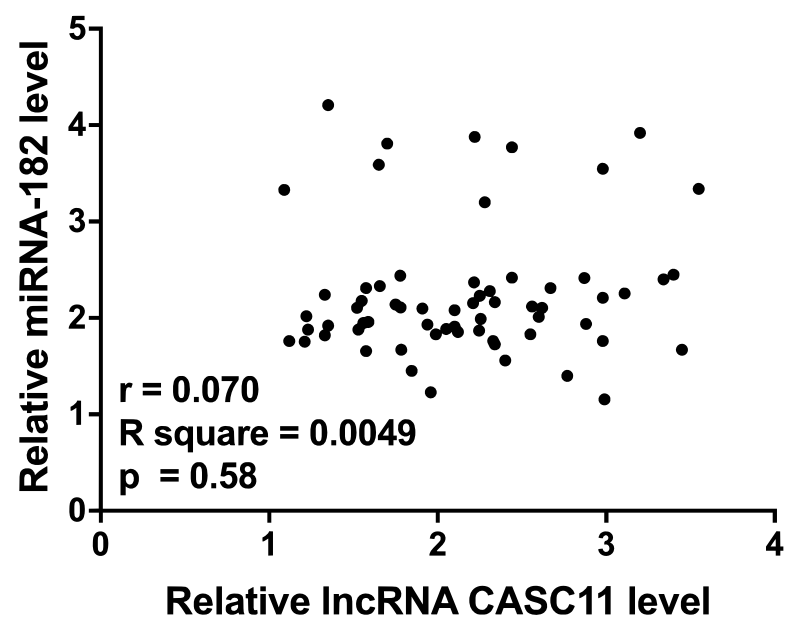

Figure I Expression levels of CASCII and miRNA-I82 were upregulated in tumor tissues and positively correlated. Results of RT-qPCR showed that expression levels of CASCII (A) and miRNA-I82 (B) were significantly upregulated in OC $(n=64)$ comparing to healthy controls ( $n=64)$. Pearson's correlation coefficient showed that expression of CASCII and miRNA-I82 was positively correlated in tumor tissues (C) but not in healthy tissues (D) $\left({ }^{*} \mathrm{p}<0.05\right)$. 
correlated (Figure 1C). In healthy tissues, CASC11 and miRNA-182 were not significantly correlated (Figure 1D).

\section{Plasma CASCII Was Upregulated in OC} Patients and Predicted Early Stage OC

Results of RT-qPCR showed that expression levels of plasma CASC11 were significantly upregulated in OC patients comparing to healthy controls (Figure $2 \mathrm{~A}, \mathrm{p}<0.05$ ). Our study included 8 cases of patient at stage I and 9 at stage II, which were early-stage OC. Potentials of CASC11 in the diagnosis of early-stage OC were analyzed by ROC curve analysis. In the ROC curve, true positive early-stage (I and II) cases were OC patients and true negative cases were healthy controls. Results showed that the area under the curve was 0.88 (standard error: $0.045 ; 95 \%$ confidence interval: $0.79-0.97$ ).

\section{High Plasma Levels of CASCII Were Closely Correlated with Poor Survival Conditions of OC Patients}

Survival curve analysis was performed using the methods aforementioned. Survival curves were compared by log-rank test. As shown in Figure 3, the overall survival rate of patients with high CASC11 level was significantly lower than that of patients with low CASC11 level within a 5-year follow-up.

\section{Overexpression of CASCII Mediated the Upregulation of miRNA-I82 in Cells of OC Cell Lines}

Overexpression experiments were performed to further explore the interactions between CASC11 and miRNA-182 in OC cells. Comparing to control (C) and negative control (NC), CASC11 overexpression led to significantly upregulated expression of miRNA-182 (Figure 4A, $\mathrm{p}<0.05$ ). In contrast, overexpression of miRNA-182 did not significantly affect CASC11 expression (Figure 4B, $\mathrm{p}<0.05$ ).

CASCII and miRNA-I82

\section{Overexpression Resulted in Promoted Cancer Cell Proliferation and Inhibited Cancer Cell Apoptosis}

In vitro cell proliferation and apoptosis assays were performed to investigate the effects of CASC11 and miRNA182 overexpression on OC cell proliferation and apoptosis. Comparing to $\mathrm{C}$ and $\mathrm{NC}$ groups, CASC11 and miRNA182 overexpression led to significantly promoted proliferation (Figure $5 \mathrm{~A}$ ) and inhibited apoptosis (Figure 5B) of OC cells $(\mathrm{p}<0.05)$.

\section{Discussion}

The functionality of CASC11 as an oncogene has only been reported in colorectal cancer. ${ }^{11}$ We found that CASC11 is overexpressed in OC and overexpression of CASC11 has diagnostic and prognostic values for OC. In addition, we also proved that CASC11 may upregulate miRNA-182 and regulate the apoptosis and proliferation of OC cells.

Expression of a large set of lncRNAs is dysregulated in OC tissues compared with normal tissues. ${ }^{13}$ Some of the differentially expressed lncRNAs have been proven to be with oncogenic or tumor suppression functions in the development and progression of OC. ${ }^{14,15}$ We first showed

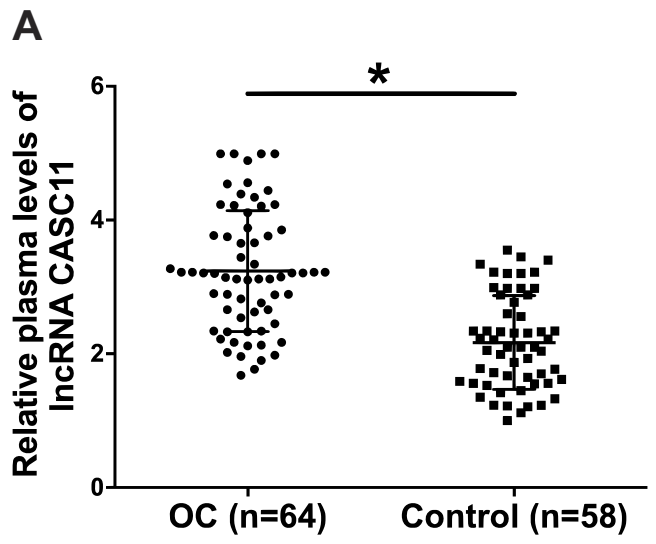

B

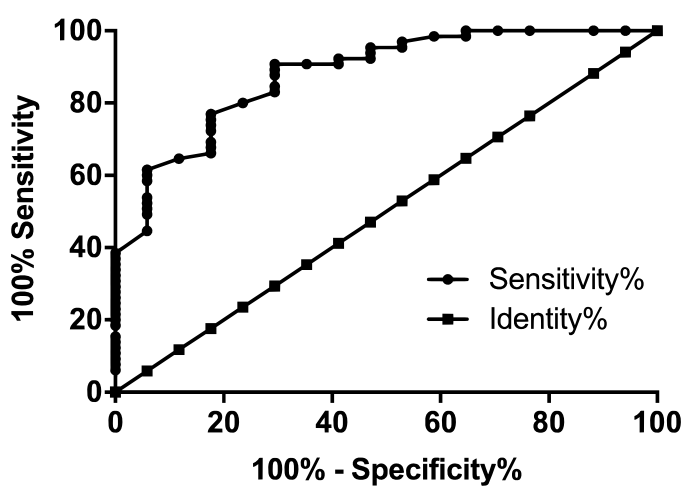

Figure 2 CASCII levels in plasma were increased in OC patients than in healthy controls and predicted early-stage OC. Results of RT-qPCR showed that expression levels of plasma CASCII were significantly upregulated in OC patients than in healthy controls $(\mathbf{A})\left({ }^{*} \mathrm{p}<0.05\right)$. ROC curve showed that increased levels of CASCII in plasma effectively separated early-stage OC patients from healthy controls (B). 


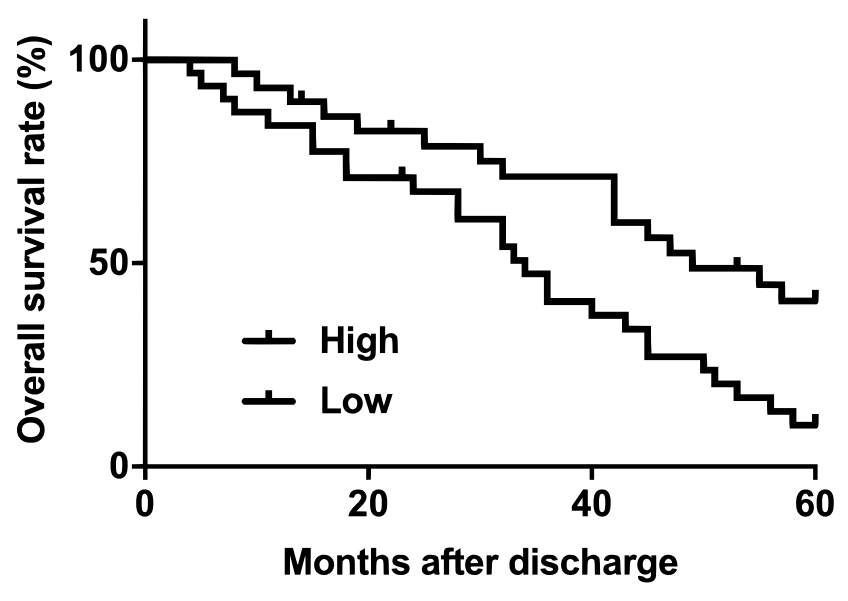

Figure 3 High plasma levels of CASCI I were closely correlated with poor survival conditions of OC patients. Survival curve comparison between high and low CASCII level groups revealed that patients $(n=34)$ with high CASCII level were with significantly worse overall survival comparing to patients $(n=30)$ with low CASCII level.

that $\mathrm{CASC} 11$ as an oncogene in colorectal cancer ${ }^{11}$ is also upregulated in OC. Our in vitro experiments using OC cell lines also proved that CASC11 overexpression may promote cancer growth by inhibiting cancer cell apoptosis and promoting cancer cell proliferation.

Most of OC patients are diagnosed at advanced stages and survival is poor. Early diagnosis is still critical for the survival of all cancer patients including OC patients. ${ }^{16}$ Therefore, the development of novel diagnostic and prognostic markers for OC is always needed. In the present study, we showed that upregulation of circulating CASC11 in plasma effectively separated early-stage OC patients from healthy controls. In addition, high levels of plasma circulating CASC11 were proven to be closely correlated with poor survival of OC patients. Therefore, CASC11 may serve as an early diagnostic marker and prognostic marker for OC. However, this hypothesis remains to be further validated by more clinical trials.

miRNA-182 plays an oncogenic role in many types of human malignancies including OC..$^{12,17,18} \mathrm{We}$ in this study confirmed the oncogenic functions of miRNA-182 in OC. In addition, CASC11 was proven to be an upstream activator of miRNA-182 in OC. However, we speculate the interaction between CASC11 and miRNA-182 is indirect due to the fact that CASC11 and miRNA-182 were only significantly correlated in $\mathrm{OC}$ tissues but not in health tissues. Therefore, certain pathological factors may mediate the interaction between them.

It is worth noting that only 8 cases of patient at stage I and 9 at stage II were included in this study to perform diagnostic analysis. Early diagnostic rate of OC is low and patients at early stages are difficult to enroll. Our future studies will try to enroll more early-stage OC patients to further confirm our conclusions. Due to technical problems, we also failed to perform CASC11 knockdown experiments to further verify its functions. Our future studies will try to include this experiment.

It is worth noting that in a recent study Shen et al reported that CASC11 is also upregulated in ovarian squamous cell carcinoma and promoted the development of chemoresistance in cancer cells. ${ }^{19}$ Therefore, CASC11 may play oncogenic roles in different types of ovary cancer.

In conclusion, CASC11 and miRNA-182 are upregulated in OC. CASC11 overexpression predicts poor prognosis and

B
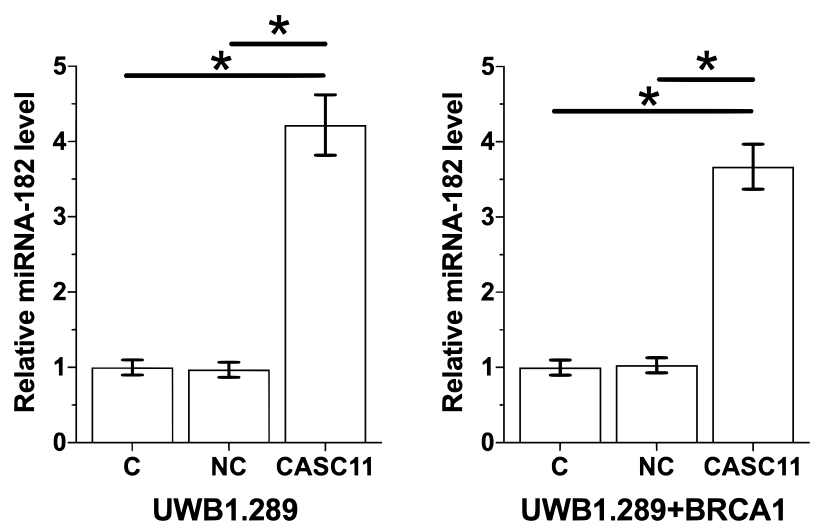

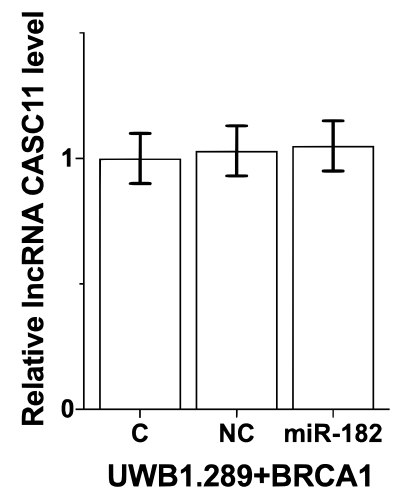

Figure 4 CASCI I overexpression mediated the upregulation of miRNA-I82 in cells of OC cell lines. CASCII overexpression led to significantly upregulated expression of miRNA-I82 (A). In contrast, overexpression of miRNA- 182 did not significantly affect CASCII expression (B). Their biological replicates were included in each experiment and mean values were presented $(* \mathrm{p}<0.05)$. 

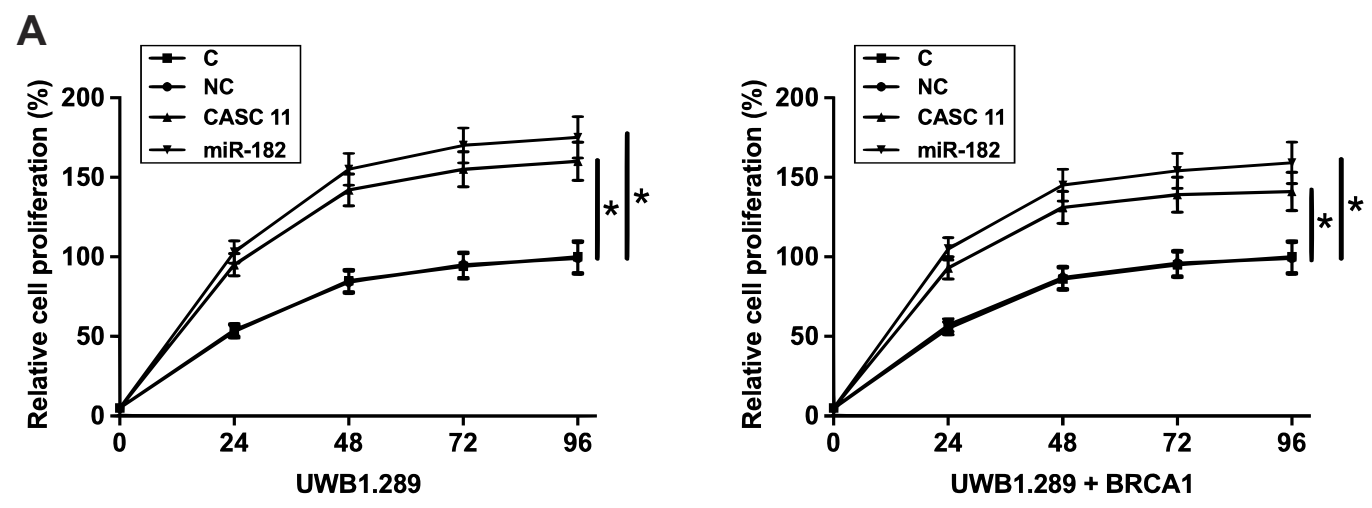

B
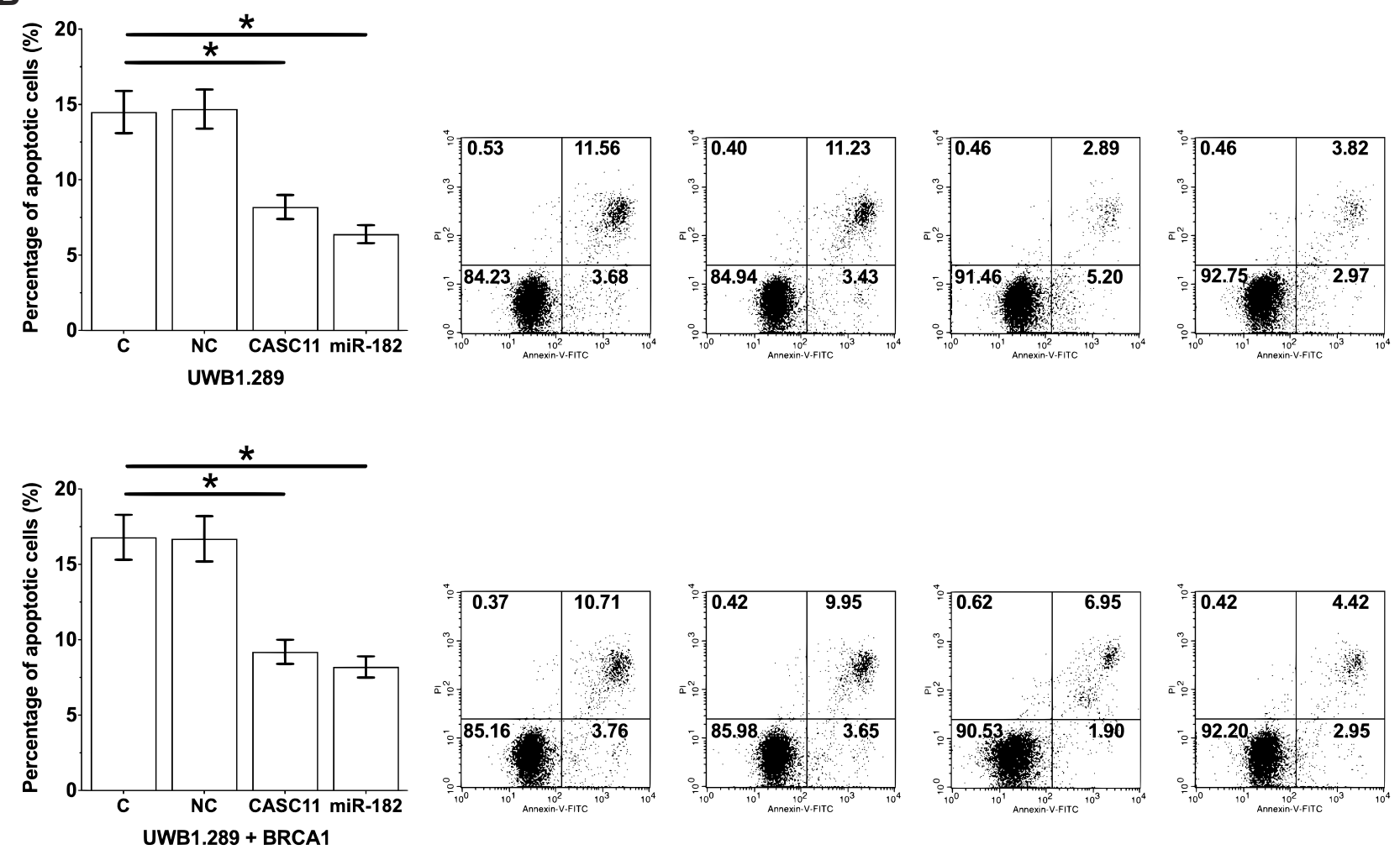

Figure 5 Overexpression of CASCII and miRNA-182 promoted cancer cell proliferation and inhibited cancer cell apoptosis. CASCII and miRNA-I82 overexpression led to significantly promoted proliferation $(\mathbf{A})$ and inhibited apoptosis $(\mathbf{B})$ of $O C$ cells. Their biological replicates were included in each experiment and mean values were presented $(* \mathrm{p}<0.05)$.

CASC11 regulates cell proliferation and apoptosis as well as microRNA-182 expression in ovarian carcinoma.

\section{Ethics Statements and Consent to Participate}

The present study was approved by the Ethics Committee of Beijing Hospital Gynecology.

\section{Data Sharing Statement}

Reasonable request only.

\section{Consent for Publication}

All patients provided consent for possible publication of this study.

\section{Author Contributions}

All authors made substantial contributions to conception and design, acquisition of data, or analysis and interpretation of data; took part in drafting the article or revising it critically for important intellectual content; gave final approval of the version to be published; and agree to be accountable for all aspects of the work. 


\section{Disclosure}

The authors report no conflicts of interest in this work.

\section{References}

1. Siegel RL, Miller KD, Jemal A. Cancer statistics, 2017. CA Cancer J Clin. 2017;67(1):7-30. doi:10.3322/caac.21387

2. Siegel RL, Miller KD, Jemal A. Cancer statistics, 2015. CA Cancer $J$ Clin. 2015;65:5-29. doi:10.3322/caac.21254

3. Simons M, Ezendam N, Bulten J, et al. Survival of patients with mucinous ovarian carcinoma and ovarian metastases: a population-based cancer registry study. Int J Gynecol Cancer. 2015;25(7):1208-1215. doi:10. 1097/IGC.0000000000000473

4. Jacobs IJ, Menon U, Ryan A, et al. Ovarian cancer screening and mortality in the UK Collaborative Trial of Ovarian Cancer Screening (UKCTOCS): a randomised controlled trial. Lancet. 2016;387 (10022):945-956. doi:10.1016/S0140-6736(15)01224-6

5. Ferlay J, Soerjomataram I, Dikshit R, et al. Cancer incidence and mortality worldwide: sources, methods and major patterns in GLOBOCAN 2012. Int $J$ Cancer. 2015;136(5):E359-E386. doi:10. $1002 / \mathrm{ijc} .29210$

6. Mercer TR, Dinger ME, Mattick JS. Long non-coding RNAs: insights into functions. Nat Rev Genet. 2009;10(3):155-159. doi:10.1038/nrg2521

7. Fatica A, Bozzoni I. Long non-coding RNAs: new players in cell differentiation and development. Nat Rev Genet. 2014;15(1):7-21. doi: $10.1038 / \operatorname{nrg} 3606$

8. Shi X, Sun M, Liu H, et al. Long non-coding RNAs: a new frontier in the study of human diseases. Cancer Lett. 2013;339(2):159-166. doi:10.1016/j.canlet.2013.06.013

9. Gibb EA, Brown CJ, Lam WL. The functional role of long non-coding RNA in human carcinomas. MMol Cancer. 2011;10 (1):38. doi:10.1186/1476-4598-10-38

10. Jalali S, Bhartiya D, Lalwani MK, et al. Systematic transcriptome wide analysis of IncRNA-miRNA interactions. PLoS One. 2013;8(2): e53823. doi:10.1371/journal.pone.0053823
11. Zhang Z, Zhou C, Chang Y, et al. Long non-coding RNA CASC11 interacts with hnRNP-K and activates the WNT/ $\beta$-catenin pathway to promote growth and metastasis in colorectal cancer. Cancer Lett. 2016;376(1):62-73. doi:10.1016/j.canlet.2016.03.022

12. Wang YQ, Guo RD, Guo RM, et al. MicroRNA-182 promotes cell growth, invasion, and chemoresistance by targeting programmed cell death 4 (PDCD4) in human ovarian carcinomas. $J$ Cell Biochem. 2013;114(7):1464-1473. doi:10.1002/jcb.24488

13. Liu SP, Yang JX, Cao DY, et al. Identification of differentially expressed long non-coding RNAs in human ovarian cancer cells with different metastatic potentials. Cancer Biol Med. 2013;10 (3):138-141. doi:10.7497/j.issn.2095-3941.2013.03.003

14. Xiu Y, Sun K, Chen X, et al. Upregulation of the lncRNA Meg3 induces autophagy to inhibit tumorigenesis and progression of epithelial ovarian carcinoma by regulating activity of ATG3. Oncotarget. 2017;8(19):31714-31725. doi:10.18632/oncotarget.v8i19

15. Chen S, Wang L, Sun K, et al. LncRNA PCGEM1 induces ovarian carcinoma tumorigenesis and progression through RhoA pathway. Cell Physiol Biochem. 2018;47(4):1578-1588. doi:10.1159/0004 90931

16. Lutz AM, Willmann JK, Drescher CW, et al. Early diagnosis of ovarian carcinoma: is a solution in sight? Radiology. 2011;259 (2):329-345. doi:10.1148/radiol.11090563

17. Wang S, Yang MH, Wang $\mathrm{XY}$, et al. Increased expression of miRNA-182 in colorectal carcinoma: an independent and tissue-specific prognostic factor. Int J Clin Exp Pathol. 2014;7 (6):3498

18. Liu H, Wang Y, Li X, et al. Expression and regulatory function of miRNA-182 in triple-negative breast cancer cells through its targeting of profilin 1. Tumour Biol. 2013;34(3):1713-1722. doi:10.1007/ s13277-013-0708-0

19. Shen F, Feng L, Zhou J, et al. Overexpression of CASC11 in ovarian squamous cell carcinoma mediates the development of cancer cell resistance to chemotherapy. Gene. 2019;710:363-366. doi:10.1016/j. gene.2019.06.011

\section{Publish your work in this journal}

Cancer Management and Research is an international, peer-reviewed open access journal focusing on cancer research and the optimal use of preventative and integrated treatment interventions to achieve improved outcomes, enhanced survival and quality of life for the cancer patient.
The manuscript management system is completely online and includes a very quick and fair peer-review system, which is all easy to use. Visit http://www.dovepress.com/testimonials.php to read real quotes from published authors. 